\title{
Neurologists don't do windows
}

\author{
John W Griffin
}

Some readers might be surprised to learn that neurology sprang from roots that were inextricably entwined with syphilis. Imagine the practice of Johns Hopkins' first neurologist, Henry Thomas, who cared for 1,200 patients with tabes dorsalis between 1888 and 1898. Happily, the boundaries of neurology have changed with time, and they continue to evolve. Only 30 years ago, neither Alzheimer's disease nor sleep disorders were thought of as core concerns of neurology. Now, both are major fields of research and clinical care. Still, like the efforts of the assertive domestic who announces, "I don't do windows," our efforts are defined as much by what we avoid as by what we do. Undoubtedly, the areas we choose to avoid are influenced by many factors, including tradition, but we should recognize that they include some major sources of neurologic morbidity.

My list of candidates for 'new' core areas of neurology 30 years from now includes cervical and lumbar spine disease, diabetic neuropathy, neuropathic pain, head injury, the neurology of infectious diseases, and the neurology of substance abuse. Few neurologists solicit the referral of patients with these problems, and surprisingly few are investigating pathogenesis or developing new treatments in these areas. Yet, all of these disorders are targets for harnessing the combination of basic and clinical science in which neurology excels.

All these conditions have enormous prevalence. Spine disease is the main cause of lost work effort in the US, and a major target for surgical intervention, but suffers from a paucity of data to drive decisions. The prevalence of substance abuse in the US requires no
The diseases

that we

consider

integral to

neurology

reflect our

training and

define what we

teach the next

generation of

neurologists.

JW Griffin is the Editor-in-Chief of Nature Clinical Practice Neurology, and Director of the Department of Neurology and Neurologist-in-Chief at Johns Hopkins University School of Medicine, Baltimore, MD, USA.

\section{Competing interests \\ The author declared he has no competing interests.}

www.nature.com/clinicalpractice doi:10.1038/ncpneuro0096 comment. Neurologists have made vital contributions to understanding the outcome of head injury, but few are involved in developing neuroprotective or axonoprotective strategies for aspects such as delayed axonal degeneration. Neuropathic pain underlies the morbidity of the most prevalent neuropathies, including diabetic neuropathy, as well as postherpetic neuralgia, complex regional pain syndrome and neuroma pain, and the pharmaceutical industry sees an enormous target for development of new treatments, but the effort involves relatively few neurologists. Diabetes is epidemic in the developed world, to the extent that the prevalence of impaired glucose tolerance has topped $15 \%$ of the population, and recent data suggest that nerve injury can begin before frank diabetes. Yet, this most prevalent of neuropathies is cared for predominantly by the primary physicians and endocrinologists, and at present neurologists are widely thought to add little to care.

I've never understood the aversion to doing windows; few things give the immediate reward of taking a dirty window to a sparkling one. Were I to found a cleaning service (admittedly a low-probability event), the motto might be 'Let us do your windows.' The diseases that we consider integral to neurology reflect our training and define what we teach the next generation of neurologists. Young neurologists who overcome the traditional aversion to fields such as the ones listed have the opportunity to have enormous impacts on health, as well as sparkling careers. This journal can contribute to the evolution of our boundaries by ensuring that relatively ignored fields are included in its reviews. 